\title{
Maladie de Chagas et autoimmunité
}

Infection parasitaire due à un trypanosome, la maladie de Chagas est caractérisée par des désordres cardiaques et neurologiques qui peuvent s'expliquer en grande partie par des mécanismes autoimmunitaires.

\section{Mireille Joskowicz Chargée de recherches à l'unité d'immunoparasitologie de l'Insti- tut Pasteur.}

\section{REFERENCES}

1. Chagas C. Nova trypanosomiase humana. Mem Inst Osmaldo Cruz 1909; 1: 159-218.

2. Krettli AU, Brener Z. Protective effects of specific antibodies in Trypanosoma cruzi infections. I Immunol 1976; 116 : 755-6o.

3. Romana C, Meyer H. Estudo do ciclo evolutivo do Schizotrypanum cruzi em cultura de tecido de embriao de gelinha. Mem Inst Oswaldo Cruz 1942 ; 37: 19-27.

\section{ADRESSE}

M. Joskowicz : unité d'immunoparasitologie Institut Pastcur, 28, ruc du Doctcur-Roux, 75724 a maladie de Chagas est une maladie parasitaire due à Trypanosoma cruzi, protozoaire flagellé transmis par des triatomidés hématophages [1]. C'est une maladie qui sévit essentiellement en Amérique Latine où elle affecte environ 10 à 12 millions de personnes. L'infection par $T$. cruzi est caractérisée par deux phases. La première phase, ou phase aiguë qui dure 1 à 2 mois est marquée par l'existence d'une parasitémie accompagnée parfois de signes généraux tels que fièvre et altération de l'état général. Durant cette phase, les trypanosomes circulent sous forme de trypomastigotes libres dans le sang, se multiplient dans les macrophages et les cellules endothéliales sous forme d'amastigotes (forme intracellulaire) qui se différencieront en trypomastigotes. La présence de parasites dans l'organisme induit la synthèse d'anticorps spécifiques anti-T. cruzi qui ne semblent pas suffisants pour éliminer le parasite de l'organisme bien qu'ils soient capables de lyser les parasites in vitro [2]. La deuxième phase de la maladie est la phase chronique qui dure toute la vie de l'individu; il n'existe pas aujourd'hui de guérison connue de la maladie de Chagas. Cette phase est caractérisée par une parasitémie très faible, détectable seulement par xénodiagnostic; les parasites sont en effet séquestrés dans les tissus de l'hôte, alors que prennent place les manifestations pathologiques comme les troubles cardiaques, qui vont de simples modifications de l'électrocardiogramme à des insuffisances cardiaques graves, et parfois à la mort subite par rupture du cœur. Les atteintes du système nerveux autonome peuvent entraîner des pathologies digestives avec dilatations organiques telles que mégaoesophage et mégacolon. La maladie peut aussi persister sous la forme asymptomatique chez de nombreux sujets infectés. Ces formes asymptomatiques posent actuellement un problème important au niveau des transfusions sanguines qui jouent un rôle potentiel considérable dans la transmission de la maladie.

\section{La maladie humaine}

L'infection de l'homme se fait principalement par les fèces contaminées des Réduvidés. Le Réduve se nourrit du sang humain par piqûre, principalement la nuit sur les régions exposées du corps : visage, avant-bras. Lors de la piqûre, l'insecte expulse ses fèces contenant des épimastigotes et des trypomastigotes métacycliques infectieux. En général, l'individu se gratte et les parasites pénètrent alors les muqueuses et atteignent les tissus sous-cutanés. D'autres voies de contamination existent : la voie transplacentaire pour le fotus, la voie parentérale lors des transfusions sanguines et plus rarement par accident au laboratoire. Dans les zones endémiques, l'infection a lieu généralement chez les enfants, où elle a un développement plus sévère que chez l'adulte. Après leur entrée dans la peau, les parasites créent une 
réaction inflammatoire locale et une lymphadénite aiguë régionale. La conjonctive oculaire est une voie de pénétration fréquente. Un œedème unilatéral apparaît avec conjonctivite et adénite pré-auriculaire (signe de Romaña).

Dans le même temps, les parasites pénètrent dans la circulation sanguine et envahissent les cellules du système réticulo-endothélial, ainsi que les cellules musculaires et gliales. La raison du choix par le parasite de l'invasion préférentielle de ces tissus n'est pas encore connue. Le parasite quitte les capillaires en perforant activement la membrane vasculaire puis la membrane plasmatique de la cellule hôte, grâce à un potentiel enzymatique lytique efficace. A l'intérieur de la cellule, le trypomastigote se transforme rapidement en forme intracellulaire appelée amastigote, forme dépourvue de flagelle. Cette forme amastigote se multiplie par division binaire à l'intérieur de la cellule, de manière asynchrone : la transformation en trypomastigotes n'intervient pas au même moment pour tous les parasites de la cellule. Lorsque le premier trypomastigote est différencié, il rompt la membrane cellulaire et rejoint la circulation sanguine, contribuant au maintien de l'infection par réinfection de nouvelles cellules. Les amastigotes non différenciés ne sont pas viables, ils restent au voisinage des cellules éclatées et dégénèrent in situ, pouvant devenir la cible de lésions inflammatoires locales [3].

Les réactions inflammatoires sont fréquentes dans la maladie de Chagas et elles ont des caractéristiques différentes suivant le stade de la maladie. Dans la phase aiguë, les infiltrats cellulaires sont petits et composés de neutrophiles, parfois d'éosinophiles. Au fur et à mesure du développement de la maladie, les monocytes et les lymphocytes apparaissent dans les réactions inflammatoires et plus tardivement les histiocytes. Il semble que chez l'homme, les infiltrats aient été localisés autour des cellules éclatées, au voisinage des amastigotes dégénérés. Dans la phase tardive de la maladie, les foyers inflammatoires peuvent confluer et la présence de grandes cellules histio-monocytaires contri-



Venu d'une petite ferme du Minas Gerais, Carlos Chagas entre à l'Ecole de Médecine de Rio de Janeiro au moment où une complète rénovation des études médicales avait lieu. Grâce à l'aide d'un de ses professeurs, Miguel Couto, il participe à l'examen post-mortem de nombreux malades et acquiert là une connaissance admirable des mystères de l'agression du corps humain par les maladies. En même temps, pour améliorer ses ressources, il donne des cours sur la Malaria.

A cette époque, la médecine au Brésil vit un moment extraordinaire avec l'éradication de la fièvre jaune du littoral brésilien grâce aux travaux d'Oswaldo Cruz. Sa notoriété conduit à l'établissement d'un grand institut pour la Recherche Médicale : l'Institut Oswaldo Cruz de Rio de Janeiro. Les étudiants les plus brillants du Brésil y sont attirés et parmi eux Carlos Chagas. Pour sa thèse, il choisit l'étude de la Malaria et présente en 1903 un mémoire sur " études hématologiques dans le paludisme ". $\mathrm{La}$ malaria est très répandue au Brésil à cette époque, et Carlos Chagas est amené à conduire plusieurs campagnes contre la malaria, travaillant en compa- gnie de savants comme Hartmann, parasitologue, Prowazek, Dürke et Giemsa, pathologistes. C'est au cours d'une expédition dans l'arrière-pays à l'occasion de la construction d'une voie ferrée Rio-Belem qu'il découvrira la maladie qui porte désormais son nom. Ayant atteint le nord de l'état du Minas Gerais près de la rivière San Francisco, les travaux doivent être arrêtés à cause d'une épidémie très grave de paludisme. Installé dans un wagon de chemin de fer près du petit village de Lassance, Carlos Chagas se met à examiner systématiquement le sang des villageois. C'est là en 1909 qu'il découvrit le trypanosome qui fut nommé Schizotrypanum d'abord puis Trypanosoma Cruzi en l'honneur d'Oswaldo Cruz.

Carlos Chagas établit le cycle du parasite, découvre le vecteur et décrit la pathologie de la maladie de Chagas. Cette découverte a un retentissement très important à l'Académie Nationale de Médecine au Brésil, et aussi à l'étranger. Cependant, malgré le prestige acquis par Carlos Chagas du fait de sa découverte, une controverse éclate au sein du monde médical brésilien, mettant en doute l'existence de la trypanosomiase américaine. Pendant une année (1923), la querelle ébranle le milieu médical et attire l'attention de l'Académie jusqu'à la reconnaissance définitive de la découverte de Carlos Chagas. Le prestige de Carlos Chagas en est toutefois ébranlé et malgré la création de la Chaire de Médecine Tropicale à l'Ecole de Médecine de Rio (1926) qu'il occupe jusqu'à sa mort (1934), l'enseignement de la maladie de Chagas sera marginalisé jusqu'en 1935. A côté de ses travaux de parasitologue et pathologiste, Carlos Chagas marque très profondément la vie médicale de son pays en réorganisant complètement les services de Santé Publique du Brésil. 
bue à la formation de granulomes inflammatoires. Pour Köberlé [4], il est clair que les réactions inflammatoires résultent de la présence du parasite; cependant les produits de dégradation du parasite seraient plus en cause que le parasite luimême. Au stade chronique, l'évolution d'une myocardite sévère ne peut être reliée au parasitisme des cellules musculaires cardiaques. A ce stade, les parasites intratissulaires sont rares, non décelables morphologiquement. Dans la pathologie nerveuse, Köberlé a montré que le nombre des cellules des ganglions sympathiques est très diminué chez l'homme atteint d'infection chronique à T. cruzi. Des mécanismes autoimmuns sont probablement impliqués dans le développement des lésions tardives de la maladie de Chagas, sans qu'on puisse, à l'heure actuelle, attribuer un rôle prépondérant aux cellules cytotoxiques ou aux auto-anticorps.

\section{Les Iymphocytes}

Depuis une dizaine d'années, plusieurs laboratoires se sont attachés à montrer le rôle des lymphocytes dans la pathologie chagasique, en particulier des lymphocytes cytotoxiques pour les tissus de l'hôte. En 1974, Santos-Buch et al ont montré que des lymphocytes de lapins infectés chroniquement par $T$. cruzi sont capables de lyser des cellules cardiaques de lapins parasités ou non [5]. Un antigène à réactivité croisée entre une fraction de $T$. cruzi et des cellules de cœur a été mis en évidence dans un test d'inhibition du macrophage. Des lymphocytes de malades humains, étudiés en test d'inhibition de la migration des macrophages, ont donné une réaction positive vis-à-vis d'antigènes de myocarde. Par ailleurs, des cellules $\mathrm{T}$ cytotoxiques vis-à-vis de cellules de cour fétal humain parasitées ou non sont présentes chez des malades chagasiques [6]. La capacité lytique de ces cellules est beaucoup plus importante chez des malades en phase aiguë que chez des malades en phase chronique. De tels résultats suggèrent que les cellules $T$ cytotoxiques jouent un rôle au cours de la phase aiguë de la cytotoxiques stimulées par $T$. cruzi reconnaîtraient sur les cellules de l'hộte l'antigène à réactivité croisée déclenchant le phénomène de lyse cellulaire. Dans le cadre des réactions à médiation cellulaire, Montufar a étudié les réactions d'hypersensibilité retardée (HSR) à l'antigène $T$. cruzi chez vingt malades en phase chronique de maladie de Chagas [7]. $100 \%$ des malades avaient une réaction positive contre $20 \%$ des contrôles. La présence de cellules spécifiques de $T$. cruzi capables de faire une réaction d'HSR chez l'homme pourrait être rapprochée des nombreux infiltrats inflammatoires caractéristiques de la phase chronique de la maladie. L'étude, par immunocytochimie, des marqueurs de surface des cellules composant ces infiltrats, permettra leur caractérisation in situ. De même, l'utilisation d'anticorps monoclonaux anti- $T$. cruzi bien définis permettra de répondre à la question de la présence de parasites ou fractions parasitaires au centre des foyers inflammatoires.

\section{Autoimmunité humorale}

Le syndrome autoimmun de la maladie de Chagas comprend aussi des facteurs humoraux tels que la présence d'auto-anticorps chez les malades. Deux types d'autoanticorps retiennent plus particulièrement notre attention : les anticorps anti-EVI et les anticorps anti-tissu nerveux. Les anticorps anti-EVI réagissent avec la membrane plasmique des muscles striés et les cellules endothéliales; ils sont retrouvés chez $95 \%$ des malades chagasiques ayant une myocardite et chez $45 \%$ des malades asymptomatiques $[8,9]$. Les anticorps antitissu nerveux décrits par Khoury et al sont présents de manière tout à fait parallèle chez les porteurs d'anticorps anti-EVI [10]. Dans les deux cas, l'absorption des sérums sur des épimastigotes de T.cruzi inhibe leur capacité à reconnaître soit le muscle strié soit le tissu nerveux (système nerveux périphérique). Ces résultats suggèrent l'existence d'une réactivité croisée entre les tissus de l'hôte et $T$. cruzi. Récemment, un anticorps monoclonal pré- paré contre des neurones a permis de détecter une protéine de $87 \mathrm{KD}$ sur la membrane des épimastigotes de $T$. cruzi et sur les trypomastigotes de culture [11]. Ces antigènes communs supposés (EVI, neurones) seraient-ils le stimulus de réactions immunitaires anti-soi dont les auto-anticorps et les cellules cytotoxiques anti-tissus seraient les manifestations ou sont-ils seulement le signe secondaire de la destruction des cellules de l'hôte [12]? Celles-ci deviendraient alors du soi modifié et seraient reconnues par le système immunitaire comme du non-soi. Actuellement, le rôle prépondérant des auto-anticorps dans la pathologie de la maladie de Chagas reste à démontrer.

\section{Le modèle expérimental}

Pour l'étude du rôle des autoanticorps comme pour celui des cellules $T$, il faut un modèle animal correct permettant une bonne reproductibilité de la maladie, un développement assez court, et une connaissance suffisante du système immunitaire de l'animal.

Teixeira et al ont reproduit, chez le lapin, par une seule injection de trypomastigotes des lésions cardiaques tout à fait similaires à la myocardite décrite chez le malade chronique. Des cellules cytotoxiques anti-muscle cardiaque ont été mises en évidence [13]. Malheureusement la caractérisation des cellules du système immunitaire chez le lapin est pour l'instant impossible. L'utilisation de ce modèle ne semble pas avoir été poursuivie. Actuellement, à Rio de Janeiro, l'équipe du Dr Galvao développe un modèle canin pour l'étude de la myocardite, qui permettra une étude clinique fine du développement de la pathologie cardiaque.

La souris a été souvent considérée comme un mauvais modèle pour l'étude de la pathologie de la maladie de Chagas à cause de la difficulté d'exploration du cœur, des fibres et des ganglions du système nerveux autonome. Cependant, nous avons pu observer que la souris infectée par T. cruzi développe les deux phases caractéristiques de la maladie : la phase aiguë 
avec une parasitémie plus ou moins élevée qui dure environ deux mois (figures $I$ a et $I b$ ) et la phase chronique qui dure toute la vie de l'animal avec persistance à très bas niveau des parasites dans les tissus.

La phase chronique, chez la souris - qu'èlle soit sensible à $T$. cruzi comme la souche $\mathrm{C}_{3} \mathrm{H} / \mathrm{HeJ}$ ou résistante comme la souche $\mathrm{C}_{57} / \mathrm{Bl6}$ présente une pathologie caractéristique avec de nombreux foyers inflammatoires dans le cœur, les muscles et le système nerveux, très similaires à ceux observés chez les humains en phase chronique. En particulier, au cours de l'infection chronique de la souris par la souche CL de $T$. cruzi, Saïd a décrit des lésions du système nerveux périphérique semblables à celles récemment décrites chez l'homme [14-16]. Des

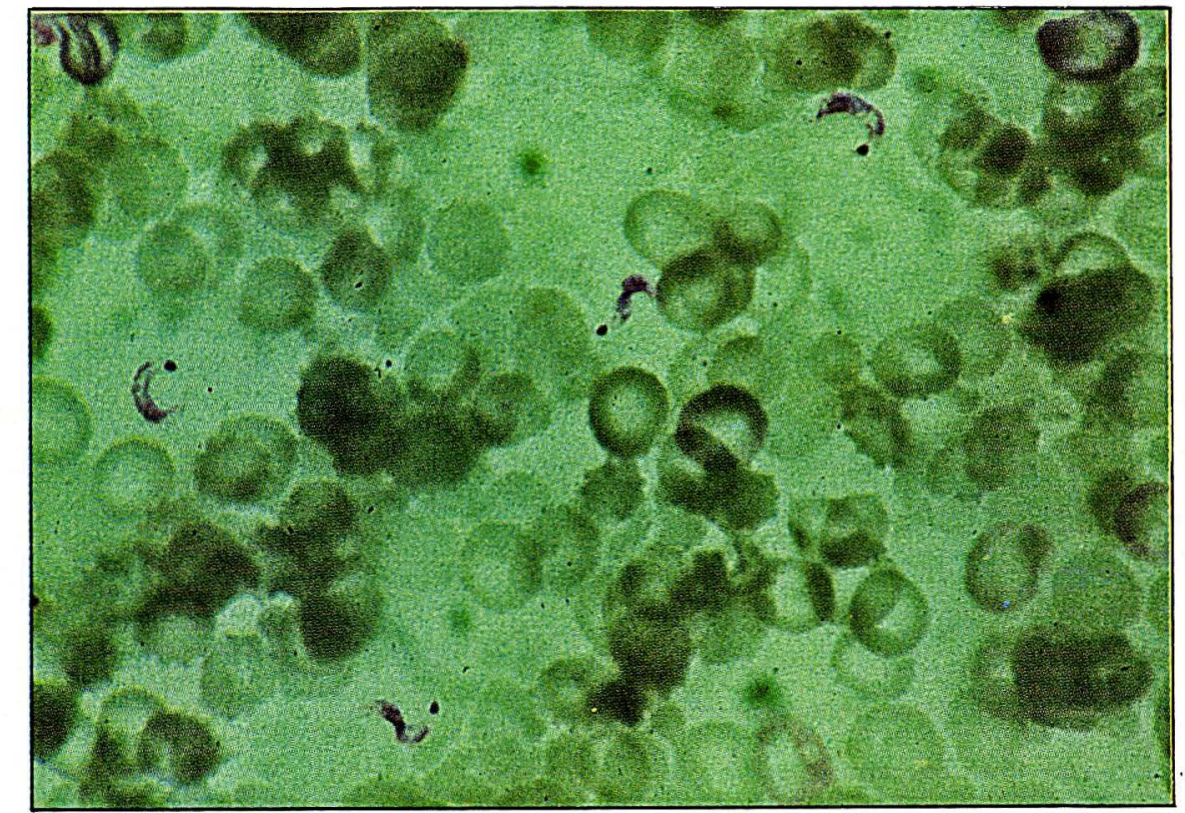

a $\Delta$

Figure 1. Phase aiguë de l'infection de la souris par Trypanosoma cruzi. a Trypomastigote sous forme libre dans le sang (phase de parasitémie).

b Amastigotes (absence de flagelle) et promastigotes (flagelle) se multipliant dans le muscle cardiaque, sans réaction inflammatoire.

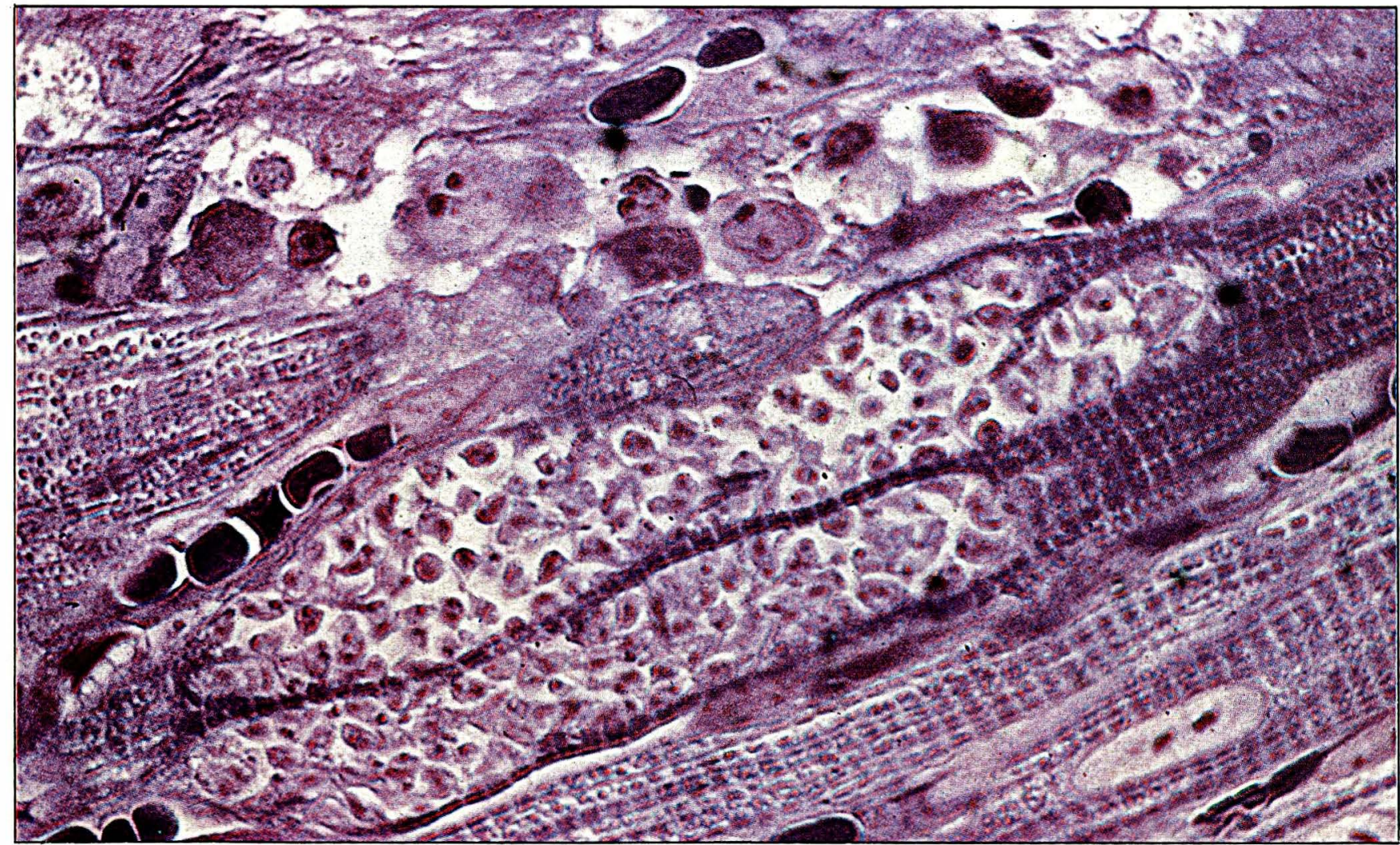




\section{REFERENCES}

4. Köberlé F. Chagas' disease and Chagas' syndromes: The pathology of American Trypanosomiasis. Adv Parasitol 1968; 6: 63-116.

5. Santos-Buch CA, Teixeira ARL. The immunology of experimental Chagas' disease. III. Rejection of allogeneic heart cells in vitro. 7 Exp Med 1974; 140: 38-53.

6. Teixeira ARL, Tcixeira G, Macedo V, Prata A. Trypanosoma cruzi-sensitized $T$ lymphocyte mediated ${ }^{51} \mathrm{Cr}$ relcase from human heart cells in Chagas' discasc. Am $\mathcal{J}$ Trop Med Hyg 1978; 27 : 1097-107.

7. Montufar OMB, Musatti CC, Mendes E, Mendes NF. Cellular immunity in chronic Chagas' discase. I Clin Microbiol $1977 ; 5$ : 401-4.

8. Cossio PM, Diez C, Szarfman A, Krentzer E, Candiolo B, Anara RM. Chagasic cardiopathy. Demonstration of a serum gammaglobulin factor which reacts with endocardium and vascular structures. Circulation 1974; 49: 13-21.

9. Cossio PM, Laguens RP, Diez C, Szarfman A, Segal A, Arana RM. Chagasic cardiopathy. Antibodics reacting the plasma membrane of striated muscle and endothelial cells. Circulation 1974; 50: $1252-9$.

10. Khoury EL, Ritacco V, Cossio PM, et al. Circulating antibodics to peripheral nerve in American trypanosomiasis (Chagas' disease). Clin Exp Immunol 1979; 36: 8-15.

11. Wood JN, Hudson L, Jessell TM, Yamamoto $M$. A monoclonal antibody defining antigenic determinants on subpopulations of mammalian neurones and Trypanosoma cruzi parasites. Nature 1982; 296: 34-8.

12. Ribciro dos Santos R, Hudson L. Trypanosoma cruzi: immunological consequences of parasite modification of host cells. Clin Exp Immunol 1980; 40: 36-41.

13. Tcixcira ARL, Tcixeira L, Santos-Buch CA. The immunology of experimental Chagas' disease. IV. Production of lesions in rabbits similar to those of chronic Chagas' disease in man. $\mathrm{Am}$ granulomes inflammatoires accompagnés de démyélinisation sont présents tout au long du nerf sciatique (figure 2), et, bien que des parasites aient pu être parfois mis en évidence dans les nerfs sciatiques de souris, à la phase aiguë d'infection, la détection avec des anticorps polyclonaux de parasites dans les lésions de la phase chronique, quelque soit le tissu considéré (nerf, cœur, muscle ou foie), n'est que très rarement positive.

L'image histologique des granulomes inflammatoires est très semblable à celle observée dans les réactions d'hypersensibilité retardée : présence de macrophages, de mastocytes et d'un important infiltrat de cellules histio-monocytaires. A la suite de Köberlé et d'autres, nous avons fait l'hypothèse que les manifestations pathologiques de la phase chronique sont de type réaction d'hypersensibilité retardée médiée par des cellules $T$ de type $T$ helper ou $T_{H S R}$. Pour élucider le rôle des cellules $T_{H S R}$ dans le développement de la pathologie, nous avons purifié les cellules $\mathrm{T}, \mathrm{L}_{3} \mathrm{~T}_{4}{ }^{+}$, Lyt $2^{-}$de souris infectées en phase chronique et les avons inoculées, par voie intraveineuse, à des souris naïves. Ces souris, sacrifiées une semaine plus tard, ont développé dans le foie, et pour certaines au niveau du nerf sciatique, de très importants foyers inflammatoires de même type que ceux observés chez les souris inf ectées chroniques. Pour essayer de définir leur spécificité, les cellules $\mathrm{T}$ de souris infectées en phase chronique par la souche CL ont été mises en culture soit en présence d'antigène $T$. cruzi (extraits de trypomastigotes) soit en présence de nerf (extraits de nerf sciatique de souris). Des lignées de cellules $T$, $\mathrm{L}_{3} \mathrm{~T}_{4}{ }^{+}$, ont été obtenues. Tout d'abord ces cellules induisent, en 48 heures après leur injection intra-neurale chez la souris, des granulomes inflammatoires ayant les mêmes caractéristiques que ceux de la phase chronique d'infection. Dans les mêmes conditions, des cellules $T$ activées in vitro par la concanavaline $A$ ne déclenchent aucune réaction. De plus, ces lignées cellulaires sont capables de transférer une réaction d'hypersensibilité retardée chez la souris naïve lorsqu'elles sont injectées dans le coussinet plantaire en même temps qu'une dose sub-inflammatoire de l'antigène utilisé in vitro pour leur propagation. Nous avons montré le rôle des cellules $T_{H S R}$ dans la pathologie chagasique. Cependant, le problème de la spécificité de ces cellules n'est pas résolu. En effet, des résultats positifs de transfert ont été obtenus en présence de l'antigène réciproque, c'est-à-dire que les lignées de cellules $T$ propagées en présence de $T$. cruzi transfèrent également une réaction d'HSR en présence de l'extrait de nerf. Nous retrouvons dans ce résultat l'indication d'une possible réactivité croisée entre $T$. cruzi et le tissu de l'hôte (ici le nerf périphérique). L'obtention d'anticorps monoclonaux contre différentes fractions de $T$. cruzi et leur étude systématique sur des tissus de mammifères devraient conduire à la détermination de l'épitope commun. Par ailleurs, des résultats tout à fait préliminaires suggèrent que dans ces lignées $T$, il pourrait exister, à côté de cellules spécifiques d'antigène, des cellules auto-réactives se développant indépendamment d'une reconnaissance antigénique spécifique. L'obtention de clones nous permettra de vérifier cette hypothèse.

Quelle que soit la réponse aux questions posées : réactivité croisée entre $T$. cruzi et les tissus de l'hôte ou auto-réactivité, il nous apparaît tout à fait intéressant de revenir sur les perturbations du système immunitaire observées chez la souris lors de la phase aiguë. Très vite après l'injection des parasites, des modifications quantitatives importantes, caractéristiques du syndrome autoimmun, prennent place dans les différents compartiments immunitaires. Ces modifications quantitatives correspondent tout à fait à celles définies par Steinberg [17] et sont très similaires à celles observées chez les différentes souches de souris NZB, BXSB, MRL à mutation lpr. Ainsi, dès le $3^{\mathrm{e}}$ jour après l'infection des souris, une forte diminution de la production d'interleukine 2 (IL 2) est observée, parallèlement à une diminution de la réponse des cellules $\mathrm{T}$ aux mitogènes in vitro et à une immunosuppression générale des réponses 
Figure 2. Réactions inflammatoires dans le nerf sciatique de souris au cours de l'infection chronique par la souche $C L$ de Trypanosoma cruzi.

(a) Préparation de fibres nerveuses $d u$ nerf sciatique par la méthode $d u$ "teasing". Fixation au tétraoxyde d'osmium. $G$ : important infiltrat granulomateux près d'un vaisseau sanguin $V$. $A$ : fibre nerveuse démyélinisée. Ce cliché sera publié dans Annals of Neuro$\log y\left[{ }_{15}\right]$.

(b) Coupe longitudinale d'un nerf sciatique $(\mathrm{I} \mu \mathrm{m})$. D: fibres nerveuses démyélinisées. $M$ : macrophage phagocytant des débris de myéline.

(c) Coupe transversale d'un nerf sciatique $(\mathrm{I} \mu \mathrm{m}) \mathrm{D}$ : fibres nerveuses démyélinisées. I : cellules inflammatoires.
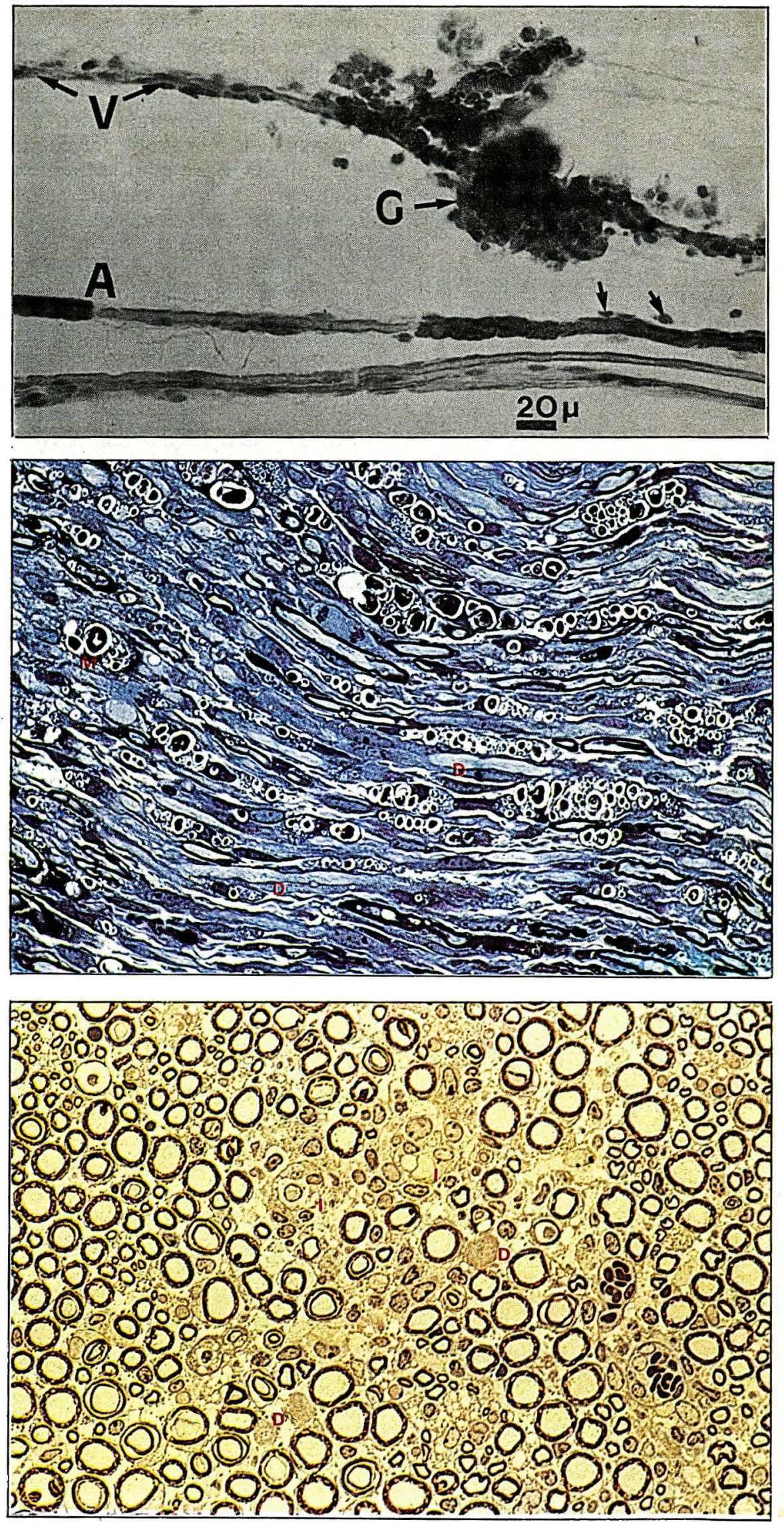


\section{REFERENCES}

14. Barreira Antunes A, Saïd G, Krettli AU Multifocal demyelinative lesions of peripheral nerves in experimental chronic Chagas' disease. Trans $R$ Soc Trop Med Hyg 1981; 75: 751 .

15. Saïd G, Joskowicz M, Barreira Antunes A, Eisen $\mathrm{H}$. Neuropathy associated with experimental Chagas' disease. Ann Neurol (sous presse).

16. Palmieri JR, LaChance MA, Conner DH. Parasitic infection of the peripheral nervous system. In: Dyck PJ, Thomas PK, Lambert EH, Bunge R, eds. Peripheral Neuropathy, vol. 2. Philadelphia: Saunders, $1984:$ 1988-2009.

17. Smith HR, Steinberg AD. Autoimmunity-a perspective. Annu Rev Immunol 1983 ; 1: 175-2 10.

18. Harel-Bellan A, Joskowicz M, Fradelizi D, Eisen $H$. Modification of $T$ cell proliferation and interleukin 2 production in mice infected with Trypanosoma cruzi. Proc Natl Acad Sci USA 1983; 80: 3466-9.

19. Ramos C, Lamoyi E, Feoli M, Rodriguez M, Perez M, Ortiz-Ortiz L. Trypanosoma cruzi: immunosuppressed response to different antigens in the infected mouse. Exp Parasitol 1978; 45: $190-9$.

20. Rowland E, Kuhn R. Suppression of cellular responses in mice during Trypanosoma cruzi infections. Infect Immun 1978 ; 20: 393-7.

21. Cunningham DS, Kuhn R. Trypanosoma cruzi-induced suppression of the primary immune response in murine cell cultures to $\mathrm{T}$-cell dependent and independent antigens. $\mathcal{J}$ Parasitol 1980 ; 66: 16-27.

22. Ortiz-Ortiz L, Parks DE, Rodriguez M, Weigle WO. Polyclonal B lymphocyte activation during Trypanosoma infection. I Immunol 1980; 124: 121-6.

23. D'Imperio Lima MR, Joskowicz $M$, Coutinho A, Kipnis T, Eisen H. Very large and isotypically atypical polyclonal plaque-forming cell responses in mice inf ected with Trypanosoma aux antigènes spécifiques [18-21]. A u cours de la phase aiguë, la majorité des cellules $T$ et des cellules $B$ sont activées sous forme blastique (Minoprio P., communication personnelle). L'activation polyclonale des cellules $B$ se traduit par une augmentation très importante du nombre de cellules productrices d'anticorps [22, 23]. Ce dérèglement du système immunitaire atteignant la plupart des cellules compétentes peut-il être à l'origine du déclenchement du processus autoimmun dans la maladie de Chagas? $\mathrm{Si}$ nous n'avons pas encore la réponse, nous pouvons toutefois imaginer que l'activation polyclonale des cellules $T$ engendrerait la prolifération de cellules anti-soi qui déborderaient le système de régulation homéostasique de reconnaissance du soi et du non-soi, alors même que l'activation polyclonale des cellules $B$ engendrerait des cellules capables de synthétiser des auto-anticorps en grande quantité.

En conclusion, nous pouvons considérer à l'heure actuelle que la maladie de Chagas a des caractéristiques générales de maladie autoimmune : dérèglement quantitatif important des fonctions cellulaires $T$ et $B$ avec activation polyclonale d'une part et suppression de la réponse aux antigènes d'autre part, production d'auto-anticorps et réaction immunitaire à médiation cellulaire anti-soi (cytotoxicité et HSR anti-tissu de l'hôte). Le rôle du parasite semble indiscutable dans le déclenchement de ce syndrome. Jusqu'à maintenant, la pathologie n'a jamais pu être reproduite par injection de sérums de phase chronique ou de phase aiguë, riches en auto-anticorps. Par contre, le rôle des cellules $T$ dans le développement de la pathologie de la phase chronique semble établi. Une question importante du point de vue de la stratégie thérapeutique reste le rôle joué par la phase aiguë pour le développement ultérieur de la pathologie, ainsi que le rôle du parasite dans l'entretien de la phase chronique. L'espoir est mis aujourd'hui dans l'utilisation par voie parentérale d'anticorps monoclonaux anti-marqueurs de surface des cellules $\mathrm{T}$ - en particulier des cellules $T$ helper - pour essayer d'arrêter leur multiplication

\section{Summary}

Chagas' disease, caused by Trypanosoma cruzi, is transmitted by blood-sucking vectors. It is endemic in South and Central America where it affects millions of people. Chagas' disease is developing in two stages: the acute phase lasting 1 to 2 months is characterized by parasitemia and general symptoms. It is followed by a lifelong chronic phase where the parasites are sequestered into the tissues (muscle, nerves) and where the pathology occurs. Chagasic symptoms are mainly cardiomyopathy and degeneration of the autonomous nervous system leading to megacolon and megaoesophagus. Several autoimmune disorders appear during $T$. cruzi infection: suppression of the specific response to antigens, polyclonal activation of $\mathrm{B}$ cells with production of autoantibodies (especially anti-endocardium and anti-nerves), development of cell mediated immunity against host antigens (cytotoxic $T$ cells and $T_{D_{T H}}$-delayed type hypersensitivity-cells). This autoimmune syndrome could be at the origin of the majority of the pathological damages of chronic Chagas' disease.

\section{TIRES A PART}

M. Joskowicz : unité d'immunoparasitologie, Institut Pasteur, 28, rue du Docteur-Roux, 75724 Paris cedex 15. 